\title{
Comportamento de ratos Wistar: importância do enriquecimento ambiental
}

\author{
Wistar Rats Behavior: Importance of Environmental Enrichment
}

\section{Resumo}

Introdução: A metodologia atual indicada para biotérios preconiza que os animais devem ficar confinados em um ambiente considerado padrão, composto de caixa plástica comunitária, água e ração. Atualmente, há grande preocupação com o bem-estar animal, sendo sugerido a necessidade de oferecer aos animais um ambiente com maior quantidade de estímulos sensoriais ou maior espaço físico disponível.

Objetivo: O presente estudo teve o objetivo de avaliar o comportamento de ratos criados sob condições ditas controle e compará-los com ratos criados sob enriquecimento ambiental.

Material e Métodos: Utilizaram-se ratos machos Wistar adquiridos na fase pós-desmame (21 dias), divididos em dois grupos denominados: Controle (C, criados em condições normais de bioterismo) e Experimental (E, criados sob enriquecimento ambiental), n=10/grupo, nos quais foi avaliado o comportamento após 30 ou 60 dias da chegada dos animais ao biotério da UNIMEP. Na avaliação comportamental, foram empregados o Teste de Campo Aberto (open field); Labirinto em Cruz Elevada (LCE) e o Teste de Contato Social.

Resultados: Foi observado menor exploração e maior ansiedade nos animais C30 e C60, enquanto os grupos E30 e E60 apresentaram menor ansiedade, maior exploração no campo aberto e no braço aberto do LCE. Na análise do contato social, foi verificado que os ratos E30e E60 mostraram-se mais impulsivos e reconheceram os intrusos em menor tempo, se comparado ao $\mathrm{C} 30$ e C60, indicando melhora no reconhecimento e consequente convívio. Conclusão: $\mathrm{O}$ ambiente enriquecido favoreceu a manifestação de diferenças comportamentais, indicando melhores condições para o animal e possivelmente menor estresse, condições ímpares para se obter resultados mais fidedignos em estudos voltados à experimentação animal.

Palavras-chave: Enriquecimento Ambiental. Bem-Estar Animal. Comportamento Animal.

\begin{abstract}
Introduction: The current methodology recommended for animal breeding recommends that animals should be confined in an environment considered standard, composed of community plastic box, water and feed. Currently there is great concern for animal welfare, being suggested the need to provide the animals an environment with greater number of sensory stimuli or bigger physical space available to the animals.
\end{abstract}


Objective: The objective of this study was to evaluate the behavior of rats raised under controlled conditions and to compare them with rats raised under environmental enrichment.

Materials and Methods: Male Wistar rats were acquired in the postweaning phase (21 days) divided into two groups: Control (C, raised under normal vivarium) and Experimental (E, raised under environmental enrichment), $n=10$ /group, in which the behavior was evaluated after 30 or 60 days of arrival of the animals to UNIMEP. In the behavioral evaluation the Open Field Test was used; Labyrinth in High Cross (LCE) and Social Contact Test.

Results: Lower exploration and higher anxiety were observed in the C30 and C60 animals while the E30 and E60 groups presented lower anxiety, greater exploration in the open field and in the open arm of the LCE. In the analysis of social contact, it was observed that the E30 and E60 mice were more impulsive and recognized the intruders in a shorter time, when compared to the $\mathrm{C} 30$ and $\mathrm{C} 60$, indicating improvement in the recognition and consequent conviviality. Conclusion: The enriched environment favored the manifestation of behavioral differences, indicating better conditions for the animal and possibly less stress, an odd condition to obtain more reliable results in studies focused on animal experimentation.

KeY-words: Environmental Enrichment. Animal Welfare. AniMAL BEHAVIOR.

\section{INTRODUÇÃO}

Ratos da espécie Wistar são utilizados em pesquisas experimentais por ser uma espécie de fácil manipulação, serem inteligentes e demonstrarem similaridades nos traços comportamentais que são de interesse em pesquisa científica. Todavia, é na natureza que os ratos normalmente são encontrados de forma livre, possuindo características próprias de suas espécies e manifestando seus comportamentos naturais, isto é, interagindo com a natureza, enfrentando desafios, procurando seus próprios alimentos, tornando-se assim, independentes. E por apresentarem tais comportamentos, esses animais, quando usados para fins experimentais, são acondicionados em biotérios sob condições controladas de temperatura e ciclo de luz. Cientistas e instituições de ensino e pesquisa têm demonstrado gran- de interesse no aprimoramento da Ciência dos animais de Laboratório, na busca de melhoria de qualidade de vida dos animais mantidos em confinamento e minimizando alterações que podem refletir em alterações em resultados de pesquisa. ${ }^{1,2}$

$\mathrm{O}$ enriquecimento ambiental é uma técnica de bioterismo em que os animais são expostos a um ambiente que fornece uma variedade de estímulos cognitivos, sensoriais e motores. ${ }^{3}$ Embora a natureza dos protocolos utilizados varie de maneira considerável entre os cientistas, o ambiente enriquecido é normalmente constituído por gaiolas grandes, contendo uma diversidade de objetos: escadas, túneis, esconderijos, brinquedos, uma roda de correr entre outros, de forma que esses objetos forneçam uma grande gama de oportunidades de estímulos visuais, somato-sensoriais, olfativas e motoras, com maiores possibilidades 
de interação, manipulação, exploração do ambiente, além dos benefícios da atividade física voluntária que os animais exercitam nessas condições, havendo ainda protocolos experimentais, onde se controla o número de animais nas gaiolas, potencializando assim as interações sociais. ${ }^{3,4}$

Pesquisadores têm sugerido que ratos acomodados em biotérios, ambiente enriquecido, sejam menos estressados, mais dóceis e, por conseguinte, mais fáceis de manejar, ao contrário dos animais confinados em ambiente padrão. ${ }^{1}$ Portanto, o enriquecimento ambiental promove uma maturação mais efetiva das áreas cerebrais do que em condições de isolamento, além de ser uma técnica importante, que consiste em modificações no ambiente físico e social de animais de biotério, ampliando assim a qualidade de vida desses animais, por meio da identificação e fornecimento de estímulos necessários para proporcionar o bem-estar psíquico e fisiológico, estimulando comportamentos típicos da espécie. ${ }^{5,6}$

O objetivo deste trabalho é avaliar o comportamento de ratos Wistar criados em ambiente enriquecido, comparando-os com ratos criados em ambiente padrão.

\section{MATERIAL E MÉTODOS}

Foram utilizados ratos Wistar, machos, adquiridos logo após o desmame, por intermédio da empresa ANILAB - Paulínia-SP, com 21 dias de vida, coincidindo com a fase pós-desmame e pesando $50 \pm 6 \mathrm{~g}$, os quais foram divididos em grupos com $n=10$ e denominados de Controle 30 (C30, criados em ambiente padrão durante 30 dias); Controle 60 (C60, criados em ambiente padrão durante 60 dias); Experimental 30 dias (E30, criados com enriquecimento ambiental durante 30 dias); Experimental 60 dias (E60, criados com enriquecimento ambiental durante 60 dias). Entende-se ambiente padrão o descrito nos guias de orientação/ criação e Bioterismo. ${ }^{7} \mathrm{Na}$ avaliação da massa dos animais, foi utilizado balança digital marca FRIZOLA ${ }^{\circ}$. O grupo E foi acondicionado em duas caixas padrão que foram interligadas, aumentando o espaço para o deslocamento, além da presença de objetos distribuídos pelo ambiente, como bolas, canos de PVC, objetos de plástico, bolas de borracha, rolhas, chocalhos, entre outros que possibilitaram aos animais subir, roer e, por conseguinte, se manterem estimulados. Esses agentes estimulantes foram trocados duas vezes por semana, instigando nos animais a busca pela "novidade" e além desses estímulos, foram proporcionados estímulos físicos como o manuseio e escovação de seus pelos a cada dois dias. Após o período de 30 ou 60 dias, os animais dos dois grupos experimentais foram avaliados por meio dos seguintes testes comportamentais: Teste de Campo Aberto ${ }^{8}$ (Open Field), Labirinto em Cruz elevada ${ }^{9,10}$ (LCE) e Memória de Reconhecimento Social ${ }^{11}$ (vide Figura 1). O estudo foi aprovado pela Comissão de Ética em Pesquisa (CEUA) da Universidade Metodista de Piracicaba (UNIMEP), sob parecer 04/2016. 


\section{ANÁlise Estatística: Na ComparaÇão dos dados, FOI UTILIZADO ANOVA E TESTE DE TUKEY, P<0,05.}

Figura 1. Avaliação comportamental. $A=$ Teste de campo aberto; $B=$ Labirinto em Cruz Elevada; $\mathrm{C}=$ Teste de Contato Social.

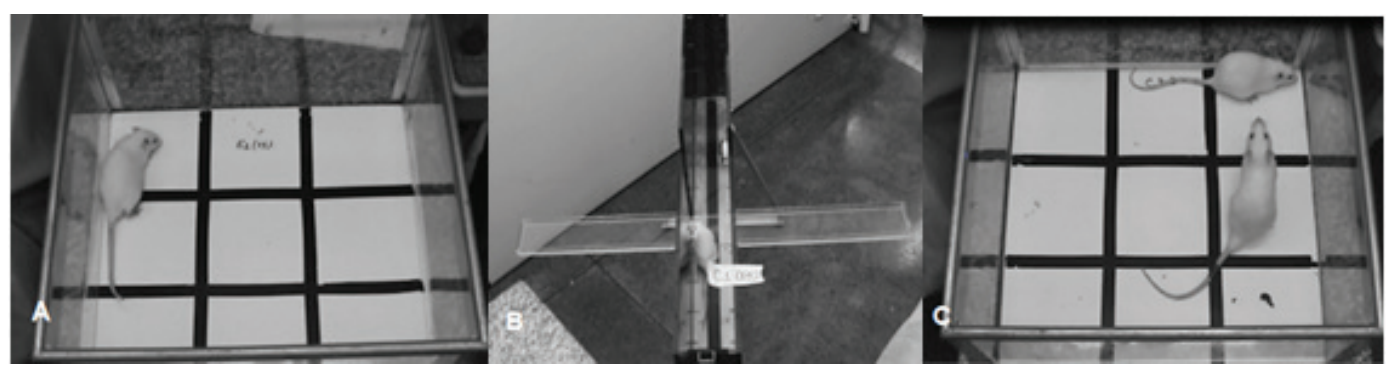

RESULTADOS E DISCUSSÃo

$\mathrm{Na}$ avaliação da massa dos animais, não foi verificado diferença entre os grupos experimentais representado por $230 \pm 20 \mathrm{~g}$ no grupo controle 30 dias (C30), $265 \pm 15 \mathrm{~g}$ no grupo controle 60 dias (C60), e $245 \pm 20 \mathrm{~g}$ no grupo experimental 30 dias (E30) e 278 $\pm 20 \mathrm{~g}$ no grupo experimental 60 dias (E60), comparado ao mesmo período de análise.

Dentro da proposta do estudo, na avaliação do comportamento no campo aberto (open field) foi observado que os grupos C30 e C60 não diferenciaram, no entanto, o grupo E30 apresentou um padrão de exploração maior se comparado ao grupo $\mathrm{C}$, atingindo índice de deslocamento $41 \%$ maior após 30 dias e 34\% após 60 dias (Figura 2). Expressaram, ainda, bipedação, atingindo valores $30 \%$ e $50 \%$ maiores se comparado ao grupo controle na mesma condição (Tabela 1). Animais criados em ambiente enriquecido manifestaram maior atividade locomotora, indicando que o ambiente foi motivador e estimulante, e sugere menor medo refletindo na atividade diferenciada dos animais. ${ }^{4}$
Além dos parâmetros descritos, a análise da posição tigmotáctica, ou seja, o tempo que o rato permanece nos cantos ou perto da periferia da arena demonstrou que enquanto os grupos C30 e C60 manifestaram ações indicativas de medo e ansiedade, os grupos E30 e E60 apresentaram índices médios $50 \%$ menores e similares, independente do período analisado, sugerindo que as condições ambientais possam ser responsáveis pelo efeito ansiolítico desenvolvido e, ainda, os animais experimentais (30 e 60) manifestaram um perfil emocional mais calmo, visto que a medida de defecação, que representa estresse, foi menor. Por sua vez, animais C manifestaram o perfil não emocional, buscando se movimentar pelas bordas do campo, além de bipedação 50\% maior, o que indica ser um animal não emocional que necessita avaliar constantemente a presença de predadores, acompanhando ${ }^{12}$ (Tabela 1 ).

Além disso, também foram analisados outros comportamentos como o grooming, que indica adaptação ao ambiente, e foi verificado que os ratos experimentais exibiram o comportamento em porcentagens $50 \%$ maiores e, dentro do caráter temporal, foi obser- 
vado que manifestaram em um tempo mais curto, indicando que se adaptam mais rápido ao meio a que estão expostos (Tabela 1).

Outro perfil que merece destaque é a avaliação do grau de estresse que os animais possam estar sendo submetidos no ambien- te indicado como padrão, pois se sabe que essa condição pode promover elevação no tempo de latência para manifestar locomoção, nesse sentido foi observado que o grupo experimental adaptou-se mais rapidamente e pôde refletir ajustes no padrão emocional.

Figura 2. Número de campos deslocados no campo aberto dos grupos controle (C) e experimental (E) avaliados nos períodos 30 e 60 dias. Os valores correspondem à média $\pm \mathrm{dp}$, $\mathrm{n}=10 .{ }^{*} \mathrm{p}<0,05$ comparado à condição controle.

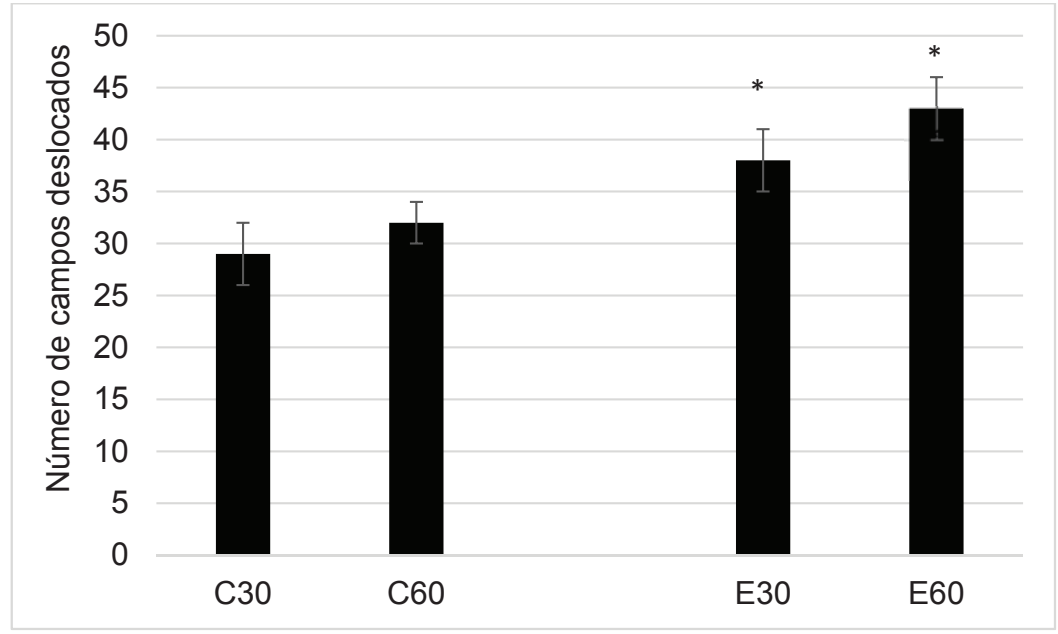

Tabela 1. Comportamento do grupo de ratos controle (C, criados em regime de normalidade de bioterismo) e experimental (E, criados sob enriquecimento ambiental) durante 30 e 60 dias de experimentação. Os valores correspondem à média $\pm \mathrm{dp}, \mathrm{n}=10 .{ }^{*} \mathrm{p}<0,05$ comparado à condição controle no mesmo período de experimentação. Alguns comportamentos estão expressos em número de vezes.

\begin{tabular}{c|c|c|c|c}
\hline Comportamento & C30 & C60 & E30 & E60 \\
\hline Bipedação & $8 \pm 2$ & $8 \pm 3$ & $4 \pm 1,2$ & $4 \pm 2,1$ \\
\hline Micção & 1 & 2 & 0 & 1 \\
\hline Defecação & 3 & 2 & 1 & 0 \\
\hline Centrotaxia & 1 & 1 & 2 & 2 \\
\hline Gromming & 1 & 1 & 2 & 2 \\
\hline Posição tigmotáctica & $8 \pm 3$ & $7 \pm 4$ & $4 \pm 1$ & $3 \pm 1$ \\
\hline
\end{tabular}




\section{Avaliação do COMPORTAMENTO NO LABIRINTO EM CRUZ ELEVADA}

A literatura mostra que no teste do labirinto em cruz elevada (LCE) ratos criados em ambiente controle de bioterismo entram menos nos braços abertos (BA), arriscando-se pouco no deslocamento até o final do $\mathrm{BA}$, prevalecendo a permanência no braço fechado (BF), indicativo de ansiedade. Esse padrão manifestado no grupo C30 não diferiu do grupo C60 (Figura 3).

A avaliação mostrou que os animais dos grupos C30 e C60 permaneceram $95 \pm 6 \%$ do tempo no $\mathrm{BF}$, não diferenciando estatisticamente do grupo $\mathrm{C} 60$, representado pela média de $90 \pm 7 \%$ no BF e $11 \pm 3 \%$ no BA. Por sua vez, o grupo E30 permaneceu $70 \pm 8 \%$ do tempo no BF e, por conseguinte, $23 \pm 4 \%$ no BA. Já o grupo E60 permaneceu $67 \pm 4 \%$ do tempo no $B F$ e, por consequência, $25 \pm 1 \%$ no BA, não diferenciando do grupo E30.

Tem-se que considerar que ao avaliar uma condição experimental, muitos fatores podem influenciar no comportamento. ${ }^{13} \mathrm{Al}-$ guns fatores estão ligados ao sujeito, como sexo, idade e estado nutricional e outros estão ligados ao procedimento experimental, como uma única ou múltiplas exposições ao labirinto. Além disso, manipulações experimentais também alteram o comportamento dos animais no teste do labirinto, como o tipo de transporte à sala de teste, o alojamento individual ou em grupo e o tempo de permanência no biotério antes do teste..$^{8,14,15}$
Quanto à causa da aversão aos BA, diferentes hipóteses buscam a explicação, como a existência de esquiva natural que os roedores apresentam em relação à novidade. Posteriormente, sugeriu-se que a aversão pelos BA resultaria do medo da altura e/ou dos espaços abertos, ${ }^{8}$ no entanto, foi proposto que a impossibilidade do animal realizar o comportamento de tigmotaxia e tendência do animal permanecer com o corpo próximo a ou em contato com superfícies verticais (peritaxia) seria o estímulo aversivo associado aos braços abertos do labirinto. Tem sido justificado esse comportamento como uma estratégia evolutiva para evitar predadores, sobretudo os predadores aéreos, fato recentemente reiterado. ${ }^{14,16,17}$

No LCE, vários fatores foram observados e merecem destaque, ou seja, os grupos $\mathrm{C}$ apresentaram exploração iniciada pelo $\mathrm{BF}$ possivelmente associado à necessidade de reconhecimento do local e localização espacial, por meio da vibração das vibrissas e mostraram baixa exploração nos BA, apontando o grau de ansiedade considerado padrão para a condição normalmente indicada para o bioterismo. Por sua vez, os animais experimentais E30 e E60 manifestaram exploração do BF $28 \%$ menor e consequente exploração 20\% maior no BA se comparado aos grupos C30 e C60, fato que aponta que os animais criados em ambiente enriquecido tiveram habilidade para analisar de forma mais primorosa os fatores de risco implicados em se locomover na alça aberta além de manifestar menor ansiedade e maior impetuosidade emocional ${ }^{18,19}$ (Figura 3). 
Figura 3. Comportamento no Labirinto em Cruz Elevada dos grupos controle (C) e experimental (E) avaliados no braço fechado (BF) ou braço aberto (BA) observados após 30 e 60 dias. Os valores correspondem à média \pm epm, $n=10 .{ }^{\star} \mathrm{P}<0,05$ comparado à condição controle.

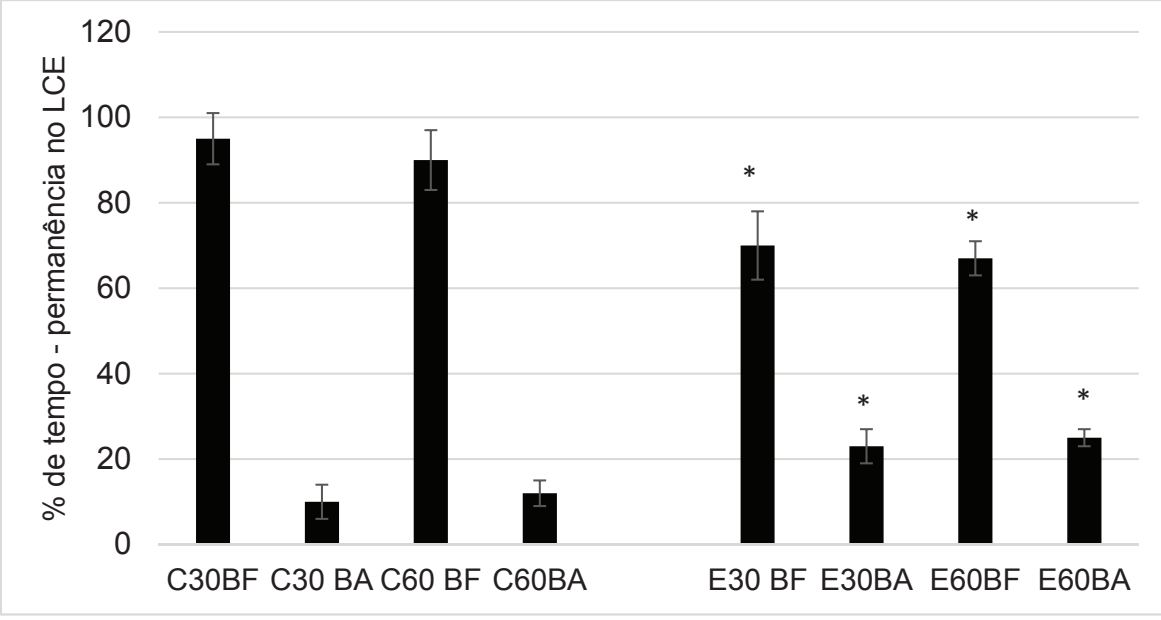

O reflexo desse comportamento indica que o ambiente forneceu uma variedade de estímulos cognitivos, sensoriais e motores como proposto. ${ }^{3}$ A base científica que dá suporte ao observado fundamenta-se nas maiores oportunidades de estímulos somatossensoriais, e motoras, permitindo a interação com o ambiente, condição que possa refletir a maturação de áreas e reconhecimento diferenciado da alça aberta.

A literatura científica tem descrito que a exposição ao ambiente enriquecido promove benefícios fisiológicos, morfológicos, bem como, ajustes na plasticidade neuronal representado pelo aumento da arborização dendrítica, gliogênese, neurogênese e modificações na expressão de genes ligados à plasticidade neuronal e, em decorrência disso, aprimoramento e melhora das respostas e pode refletir na condição comportamental diferenciada. $^{20,21}$

\section{ANÁlise do COMPORTAMENTO NO CONTATO SOCIAL}

Quando se avalia comportamento social usando como modelo experimental o rato, deve-se atentar para a atividade primorosa do sistema olfatório, o qual exerce ação primordial para a espécie. ${ }^{11} \mathrm{O}$ reconhecimento social em ratos é um instinto natural que visa investigar indivíduos novos e avalia o aprendizado e a memória, por sua vez, o comportamento de cheirar o corpo e a região anogenital, assim como perseguir o indivíduo fazem parte da investigação social. ${ }^{22} \mathrm{O}$ comportamento olfatório é acompanhado da reação de lamber e morder sendo importantes no desenvolvimento social e cognitivo. ${ }^{23}$

As análises do comportamento social do grupo C indicaram a manifestação dos comportamentos básicos, como, cheirar a região anogenital, tocar, empurrar, e lamber o ou- 
tro animal (intruso) no primeiro encontro e, em menor número, no segundo encontro realizado em curto período (15 min), acompanhando a literatura que mostra que ratos criados na condição de bioterismo, indicada na literatura, retêm até 24 h de memória olfativa. ${ }^{24,25}$ Esse comportamento não foi estatisticamente significante ao compararmos os grupos C30 e C60.

Sabe-se que no enriquecimento ambiental são potencializadas as interações sociais e o desenvolvimento da estimulação motora e sensorial. ${ }^{27,28}$ Durante a permanência nesse ambiente, os animais têm maiores possibilidades de realizar atividades diferenciadas. Dessa forma, a exposição dos animais a uma variedade de estímulos permite a manifestação dos comportamentos naturais da espécie. ${ }^{29}$ Pesquisadores têm dedicado esforços no sentido de avaliar aspectos comporta- mentais em ratos submetidos a ambientes enriquecidos e sugerem que estes são menos estressados, mais dóceis e, por conseguinte, mais fáceis de manejar se comparados a animais confinados em ambiente padrão. ${ }^{30}$

Neste estudo, foi verificado que no teste de contato social, ao introduzir o animal intruso na caixa, no segundo encontro, os animais dos grupos experimentais E30 e E60 apresentaram respostas comportamentais manifestas por olfação, aninhar-se abaixo do intruso, bem como, as demais manifestações em menor número de vezes e em menor tempo se comparado ao comportamento dos grupos C30 e C60 (Tabela 2). Um estudo demonstrou que em ambiente enriquecido há uma redução da resposta do eixo hipotalâmico-pituitário-adrenal, mediante estímulo estressante, permitindo que os animais sejam mais ativos e menos estressados..$^{30,27}$

Tabela 2. Comportamentos dos grupos experimentais de ratos Wistar. Os valores correspondem à média $\pm \mathrm{dp}, \mathrm{n}=10$ e representam o número de vezes que o comportamento foi manifestado. ${ }^{\star} \mathrm{p}<0,05$ comparado ao primeiro contato do mesmo grupo.

\begin{tabular}{|c|c|c|c|c|}
\hline No. de vezes & $\begin{array}{c}\text { C30 } \\
\left(1^{\circ} . \text { contato }\right) \\
\end{array}$ & $\begin{array}{c}\text { C30 } \\
\left(2^{\circ} . \text { contato }\right) \\
\end{array}$ & $\begin{array}{c}\text { E30 } \\
\left(1^{\circ} . \text { contato }\right) \\
\end{array}$ & $\begin{array}{c}\text { E30 } \\
\left(2^{\circ} . \text { contato }\right) \\
\end{array}$ \\
\hline Cheirar & $13 \pm 1,2$ & $6 \pm 1,0^{*}$ & $10 \pm 2$ & $4 \pm 2^{\star}$ \\
\hline Tocar & $5 \pm 2$ & $1 \pm 1^{*}$ & $6 \pm 1,1$ & $2 \pm 1,2^{*}$ \\
\hline Empurrar & 2 & $1^{\star}$ & $4 \pm 1,2$ & $3 \pm 1$ \\
\hline Lamber & $4 \pm 1$ & $2 \pm 1$ & $3 \pm 1,3$ & $3 \pm 1,1$ \\
\hline $\mathrm{N}^{\circ}$. de vezes & $\begin{array}{c}\mathrm{C60} \\
\left(1^{\circ} . \text { contato }\right) \\
\end{array}$ & $\begin{array}{c}\mathrm{C60} \\
\left(2^{\circ} . \text { contato }\right) \\
\end{array}$ & $\begin{array}{c}\text { E60 } \\
\left(1^{\circ} . \text { contato }\right)\end{array}$ & $\begin{array}{c}\text { E60 } \\
\left(2^{\circ} . \text { contato }\right) \\
\end{array}$ \\
\hline Cheirar & $10 \pm 2$ & $5 \pm 1^{\star}$ & $8 \pm 2$ & $4 \pm 1^{\star}$ \\
\hline Tocar & $4 \pm 1$ & $3 \pm 1$ & 2 & 1 \\
\hline Empurrar & 3 & 1 & 2 & 1 \\
\hline Lamber & $3 \pm 1$ & 2 & $3 \pm 1,3$ & 1 \\
\hline
\end{tabular}




\section{CONSIDERAÇõES FINAIS}

Os dados mostram a importância do enriquecimento ambiental na configuração de comportamentos diferenciados, fator básico para a qualidade de vida e redução do estresse em animais utilizados em experimentação e reitera a importância do ambiente enriquecido como metodologia primordial também a ser aplicada em Biotérios de criação de animais de laboratório, buscando linearizar a qualidade dos animais em diferentes centros de bioterismo, uma vez que os animais tornam-se menos estressados, exploram mais o ambiente e sentem menos as restrições impostas no modelo tradicional de cuidados de bioterismo.

\section{REFERÊNCIAS}

1. Garbin NLC, Faleiros RR, Lago LA. Enriquecimento ambiental em roedores utilizados para a experimentação animal: revisão da literatura. Rev. Acad. Ciênc. Agrar. Ambient. 2012, 10 (2): 153-161.

2. Pizzutto CS, Sgai MGFG, Guimarães MABV. Environmental enrichment as a tool to improve reproduction and wellbeing of captive animals. Rev Bras Reprod Anim. 2009, 33 (3): 129-138.

3. Van Praag H., Kempermann G., Gage FH. Neural consequences sequences of environmental enrichment. Nature Reviews Neuroscience. 2000, 1 (3): 191-198.

4. Mezadri TJ, Ortolan XR, Thieme AL, Piacentini N., Amaral VLL, Frajblat M. Avaliação do comportamento de ratos alojados em caixas de cores diferentes. Rev Ecologia. 2011, 10 (1): 21-26.

5. Boere V. Behavior and environmental enrichment. Biology, Medicine and Surgery of South American Wild Animals. Iowa: Iowa State Press University. In: M. E. Fowler. Z. S, Cubas, 2001.

6. Hohendorff RV. Aplicação e avaliação de enriquecimento ambiental na manutenção de bugio (Alouatta app LACÉPEDE, 1799) no Parque Zoológico de Sapucaia do Sul-RS. Dissertação de mestrado, Universidade Federal do Rio Grande do Sul, Porto Alegre, 2003.

7. Andersen ML, D’almeida V., Ko GM, Kawakami R., Martins PJF, Magalhães LE, Tufik S. Princípios Éticos e Práticos do Uso de Animais de Experimentação. UNIFESP, 2004, 167p.

8. Pellow S., Chopin P, File SE, Briley M. Validation of open: closed arm entries in the elevated plus-maze as a measure of anxiety in the rat. J Neurosci Methods. 2005; 14: 149-67.

9. Cruz APMF, Graeff FG. Ethopharmacological analysis of rat behavior on the elevaded plus-maze. Pharmacol Biochem Behav. 1994, 49: 171-176.

10. Van Praag H., Kempermann G., Gage FH. Neural consequences sequences of environmental enrichment. Nature Reviews Neuroscience. 2000, 1 (3): 191-198.

11. Moura PJ, Gimenes-júnior JA, Valentinuzzi V., Xavier G.F. Circadian phase and intertrial interval interfere with social recognition memory. Physiology \& Behavior. 2009, 96: 51-56.

12. Katz RJ, Roth KA, Carroll BJ. Acute and chronic stress effects on open field activity in the rat: implications for a model of depression. Neurosci Biobehav Rev. 1981; 5 (2): 247-51

13. Lundberg S., Abelson KSP, Nylander I., Roman E. Few long-term consequences after prolonged maternal separation in female Wistar rats. PLoS One. 2017, 21 (12): 1-12.

14. Moura PJ, Xavier GF. Memória de reconhecimento social em ratos. Rev. Psicol. USP. 2010, 21 (2): 355-389.

15. Simpson J., Kelly J. The impact of environmental enrichment in laboratory rats behavioral and neurochemical aspects. Behavioral Brain Research. 2011, 222: 246-264. 
16. Pinto WBVR, Ko GM, Valero-Lapchik V. Teste de labirinto em cruz elevada: aplicações e contribuições no estudo de doenças neuropsiquiátricas em modelos animais. RESBCAL, São Paulo. 2012, 1 (1): 102-120.

17. Bardi M., Kaufman C., Franssen C., Hyer MM, Rzucidlo A., Brown M., Tschirhart M., Lambert KG. Paper or Plastic? Exploring the Effects of Natural Enrichment on Behavioural and Neuroendocrine Responses in Long-Evans Rats. J Neuroendocrinol. 2016; 28 (5): 31-38.

18. Carola V. Evaluation of the elevated plus-maze and open-field tests for the assessment of anxiety-related behavior in mice. Behavioral Brain Research. 2002, 134: 1-2.

19. Debra AL, Gillian R B. The Ontogeny of Anxiety-Like Behavior in Rats from Adolescence to Adulthood. Rev Psychobiol. 2010, 52 (8): 731-739.

20. Würbel H. Ideal homes? Housing effects on rodent brain and behavior. Trends Neurosci, 2001; 24: 207-211.

21. Moncek F. Effects of environmental enrichment on stress related systems in rats. Journal of Neuroendocrinology. 2004, 16: 423-431.

22. Bielsky IF, Young LJ. Ocitocin, vasopressin and social recognition in mammals. Peptides. 2004, 25 (9): 156-174.

23. Kepecs A., Uchida N., Mainen ZF. The Sniff as a unit of an olfactory processing. Chemical senses. 2006; 31: 167-179.

24. Silva VC, Almeida SS. Desnutrição proteica no início da vida prejudica a memória social em ratos adultos. Rev. Nutri. 2006; 19 (2): 195-201.

25. Becker A., Grecksch G. Social memory is impaired in neonatally ibotenic acid lesioned rats. Behav Brain Res. 2000, 109 (1): 137-140.

26. Schweigert ID, Souza DOG, Perry MLS. Desnutrição, maturação do sistema nervoso central e doenças neuropsiquiátricas. Rev. Nutr. 2009; 22 (2): 17-22.

27. Segovia G., Arco A., Mora F. Environmental enrichment, prefrontal córtex, stress, and aging of the brain. Journal of Neural Transmission. 2009, 116 (8): 1.007-1.016.

28. Baumans V., Van Loo PLP. How to improve housing of laboratory animals: The possibilities of environmental refinement. Elsevier. The Veterinary Journal, 2013, 195 (1): 24-32.

29. Lambert K., Hyer M., Bardi M., Rzucidlo A., Scott S., Terhune-Cotter B., Hazelgrove A., Silva I., Kinsley C. Natural-enriched environments lead to enhanced environmental engagement and altered neurobiological resilience. J. Neuroscience. 2016, 25; 386-94.

30. Lehmann ML, Herkenham M. Environmental enrichment confers stress resiliency to social defeat through an infralimbic cortex-dependent neuroanatomical pathway. J. Neuroscience. 2011, 31: 6.159-6.173.

\section{Dados dos autores:}

\section{Pamela Gozzer}

Docente na Universidade Metodista de Piracicaba (UNIMEP). Piracicaba/SP - Brasil. pamelagozzer26@gmail.com

\section{Ellen ANDRADE}

Biologa no CTC - Centro de Tecnologia Canavieira. Piracicaba/SP - Brasil. ellen.andrade@gmail. com

\section{Patrícia Carla Paulino Belotto}

Mestranda em Ciências do Movimento Humano - CMH - FACIS - Universidade Metodista de Pira- 
cicaba (UNIMEP). Piracicaba/ SP - Brasil. pcpaulin@unimep.br

Bruno Ferreira Gonçalves Silva

Acadêmico de Medicina Veterinária - Universidade Paulista (UNIP). Campinas/SP - Brasil. brufgsilva@outlook.com

\section{André Alves Lico Mascarin}

Doutorando em Ciências do Movimento Humano. - FACIS. Universidade Metodista de Piracicaba (UNIMEP). Piracicaba/ SP - Brasil. Andre_Mascarin@hotmail.com

Carlos Alberto Silva

Docente no programa de Pós-graduação em Ciências do Movimento Humano. - FACIS. Universidade Metodista de Piracicaba (UNIMEP). Piracicaba/ SP - Brasil. casilva@unimep.br

Submetido em: 12-1-2018

Aceito em: 22-5-2018 